\title{
Disputes sur l'ergonomie de la tâche et de l'activité, ou la finalité de l'ergonomie en question
}

François Hubault et Fabrice Bourgeois

\section{Q OpenEdition}

1 Journals

Édition électronique

URL : https://journals.openedition.org/activites/1149

DOI : 10.4000/activites. 1149

ISSN : $1765-2723$

Éditeur

ARPACT - Association Recherches et Pratiques sur les ACTivités

Référence électronique

François Hubault et Fabrice Bourgeois, « Disputes sur l'ergonomie de la tâche et de l'activité, ou la finalité de l'ergonomie en question », Activités [En ligne], 1-1 | avril 2004, mis en ligne le 02 avril 2004, consulté le 21 septembre 2021. URL : http://journals.openedition.org/activites/1149 ; DOI : https:// doi.org/10.4000/activites. 1149

\section{(c) (i) (3) $\Theta$}

Activités est mis à disposition selon les termes de la licence Creative Commons Attribution - Pas d'Utilisation Commerciale - Pas de Modification 4.0 International. 


\title{
Disputes sur l'ergonomie de la tâche et de l'activité, ou la finalité de l'ergonomie en question
}

\author{
François Hubault \\ CEP Ergonomie et Écologie Humaine, Paris I Panthéon-Sorbonne \\ francois.hubault@wanadoo.fr \\ Fabrice Bourgeois \\ Ergonome consultant, Omnia intervention ergonomique \\ omnia.fb@nnx.com
}

\begin{abstract}
RÉSUMÉ
La distinction entre tâche et activité est fondatrice de l'ergonomie. Les années passant, différentes manières d'enseigner et de mettre en pratique cette distinction ont vu le jour bien sûr, et heureusement, à travers lesquelles, en fait, se sont aussi exprimées des divergences sur la finalité de l'ergonomie. Dès lors, les divergences ne sont plus seulement de détail, ou de développement, mais de fond. Rien que de normal finalement, à condition qu'elles soient discutées et visibles.

Le dernier ouvrage de Maurice de Montmollin nous offre cette occasion. Sa charge contre les sciences sociales et le reproche d'incapacité qu'il fait à l'ergonomie (et aux ergonomes) constituent un point de désaccord que nous discutons. Il nous permet de pointer des faux-semblants au sein de la communauté ergonomique, concernant la place et le sens de l'activité et le rôle des connaissances sur l'activité. Et derrière ces faux-semblants, des oppositions importantes sur la place et le rôle de l'ergonomie et des ergonomes dans la transformation du travail. Au-delà de cette "dispute », alors, mais grâce à elle, nous proposons de revisiter les contours possibles de la multidisciplinarité contemporaine de l'ergonomie.
\end{abstract}

MOTS CLÉ

Tâche, activité, intervention, démarche clinique, multidisciplinarité.

\begin{abstract}
Ergonomics is based on the distinction between task and activity. As time goes, different ways of teaching and practicing this distinction come out of course, lukyfully, which give evidence to deeper distinctions related to the very objectives of ergonomics. Thus, such important differences cannot be only said but debated, the opportunity of which is taken here when discussing Maurice de Montmollin last book. Opposing the way he charges social sciences and ergonomics (and ergonomists) with inefficiency, the authors intend to drive out pretences from the so called ergonomic community consensus about the meaning of "activity" and the knowledge of it. Behind these pretences, important oppositions on the place and the role of ergonomists in changing work conditions are discussed, and the dispute
\end{abstract}


leads to examining new frame and contents of multidisciplinarity in modern ergonomics.

\section{KEYWORDS}

Task, activity, intervention, clinic step, multidisciplinarity.

Quoiqu'on en dise, il existe au sein même de ce qu'on appelle désormais l'ergonomie centrée sur l'activité, différentes «manières de voir » et donc des manières de faire aussi qui ont pour point de départ des divergences théoriques. Dejours (1996, pp. 214-215) parle «d'écoles d'ergonomie, se distinguant par leurs postulats sur le travail, la technique et l'homme; ou mieux encore par leurs doctrines ».

Ces divergences se révèlent, par exemple, quand un étudiant de maîtrise demande un conseil sur le DESS qui lui conviendrait le mieux. Chacun peut relier telles spécificités doctrinales à telle université, mais des indications aussi vagues que «tu trouveras plutôt çà ici et plutôt d'autres choses làbas » ne suffisent pas pour orienter des étudiants sérieusement. Ces divergences se révèlent aussi, à leur façon, dans les communications présentées aux congrès de la SELF. Elles se retrouvent enfin dans la façon dont les ergonomes conçoivent les services qu'ils proposent aux entreprises ou les programmes de recherche. Chacun, ainsi, a la possibilité de retrouver et conforter par lui-même ou au sein de collectif d'affinités, ses référents doctrinaires. Pourtant, ces différences sont rarement travaillées comme tel, disputées pour les clarifier, les comprendre et les apprécier.

Cette carence trouble l'image de l'ergonomie centrée sur l'activité et handicape son développement. Notre position est que la divergence fondamentale en ergonomie porte sur le sens de l'analyse du travail. C'est bien de là, en effet, que les uns bifurquent vers l'ergonomie de la tâche alors que les autres s'engagent vers l'ergonomie de l'activité, mais la dispute sur le sens continue à l'intérieur même de l'ergonomie de l'activité qui ne constitue pas une réponse mais plutôt un changement de plan où les éléments du débats se trouvent finalement reconduits.

Telle que nous la comprenons, l'ergonomie de l'activité soutient la thèse que travailler, c'est gérer une tension issue d'une double exigence. D'une part, l'efficacité qui renvoie aux attentes de l'entreprise, ce qu'elle demande à travers la tâche et, d'autre part, la santé qui renvoie aux attentes de la personne, ce qu'elle vise à travers son engagement dans l'activité.

Travailler c'est donc répondre à un système d'exigences, tout autant économiques que subjectives.

Ce disant, l'analyse du travail n'a pas simplement en charge de décrire ce que fait l'opérateur. Elle doit aussi servir au développement de l'activité, dans l'ordre subjectif comme dans l'ordre économique. En d'autres termes, elle doit s'intéresser à ce que "on »-1'opérateur, l'entreprise, ...mais aussi, il faut bien s'en rendre compte, la société (cf. l'âge de la retraite...) - peut et veut faire du «travail ».

De fait, pour nous, il en découle alors une double exigence pour l'ergonome, celle de « répondre à... » un problème, mais aussi de "répondre de » ce qu'il fait lui-même dans l'intervention (en répondre). Mais cette reconnaissance de double exigence constitue un point de divergence, une distinction d'écoles. Et tout cela, justement, est matière à dispute.

\section{1.- Un exemple de dispute, à propos de la thèse de M. de Montmollin}

Dans son dernier ouvrage, M. de Montmollin (2002) développe une thèse que nous résumons comme suit : 
- les Sciences Humaines et Sociales (SHS) ${ }^{1}$ n'ont rien transformé de significatif dans le registre de l'organisation concrète des entreprises... (ce point est très discutable, mais nous ne le discuterons pas ici, admettons-le provisoirement) ;

- parce qu'elles ne sont pas assez scientifiques :

- elles procèdent, dit-il avec l'ironie qu'on lui connaît, de «propos d'après dîner » plutôt que « d'avant dîner », qu'on imagine plus sobres et plus sérieux...,

- elles parlent plutôt « Sur» l'organisation que «Pour» (autrement dit, elles sont plus un discours qu'une véritable contribution, une capacité concrète de transformation...),

- elles sont donc en peine de répondre au « et alors ? » que très légitimement on leur retourne ;

- de sorte que le taylorisme reste finalement indépassable, du moins indépassé...

On ne dissimulera pas le pouvoir d'interpellation de cette diatribe. De fait, tous les points du raisonnement appellent une réaction, et constituent finalement autant de rendez-vous sur lesquels se jouent, pour nous, le métier d'ergonome, la connaissance et la reconnaissance de ce qu'il produit et les passerelles entre la recherche et la pratique.

\section{Dispute sur la « scientificité » de l'ergonomie}

Commençons par ceci : nous ne croyons pas une seconde que l'échec supposé des SHS tient à leur manque de scientificité. Plus exactement, si la scientificité est en cause, c'est plutôt que les SHS $d u$ travail n'assument pas ou mal leur objet, le travail. Pour nous, les difficultés pour transformer l'organisation concrète de travail viennent de la difficulté d'assumer, autant dans l'entreprise que dans l'ergonomie, ce que travailler veut dire. Les entreprises sont trop occupées à essayer de penser leurs organisations dans les termes que leur dictent des « sciences dures » qu'elles croient encore exactes en ces matières, et qui traitent, elles, du travail comme un objet maîtrisable, réglable, homogène. Inévitablement, les formalisations auxquelles elles parviennent alors, sont pauvres. Les « résultats » ont une efficacité en termes d'amélioration des objets utilisés dans le travail - en termes d'utilisation d'objet -, mais pas vraiment en termes d'activité de travail. Les ergonomes peuvent travailler sur « ces objets», « se rendre utiles » pour les rendre plus confortables, mais s'ils se cantonnent à cela ils se détournent alors de deux réalités auxquelles ils doivent aussi répondre. La première est l'hétérogénéité du travail car le travail, c'est l'expérience de ce qui résiste, c'est la nécessité d'accorder ensemble des dimensions qui ne répondent pas aux mêmes logiques, ce en quoi, justement, le travail se distingue $\mathrm{du}$ « fonctionnement». La seconde est son hétéronomie car les raisons d'agir, les systèmes de valeur de l'entreprise et des personnes ne peuvent pas être les mêmes.

Le discours des SHS sur le travail est nécessairement moins formel que celui des sciences dures. Il n'est pas pour cela moins rigoureux si ce terme signifie qu'il se doit d'être en accord avec la nature même de l'objet que nous traitons ${ }^{2}$. La césure «avant / après dîner » paraîtrait presque sous cet angle un propos d'après dîner... Qualifier le discours des SHS de moins «scientifique » n'a de sens que dans une acception de la science qui disqualifie l'idée même de science humaine et sociale. Nous pourrions convenir de ce reproche de manque de scientificité, mais en suivant Schwartz (2000) plutôt que de Montmollin, c'est-à-dire avec l'idée qu'il faut inscrire l'activité de travail dans la perspective ergologique du concept d'activité humaine...

Pour nous, l'«échec» des SHS tient à la nature des transformations qu'exige(rait) leur « application». Les managers s'appuient sur les sciences sociales comme l'ivrogne au réverbère...

1. Il s'agit des psychologies du travail (la psychologie industrielle, la psychologie des organisations, les psychologies cliniques), de la sociologie du travail, des sciences de gestion et de l'ergonomie.

2. Notre propos est ici de principe, car une critique de la qualité scientifique des SHS reste évidemment pertinente concernant le degré d'accord entre le discours des SHS et la nature de l'objet sur lequel porte ce discours 
ironisait Eugène Enriquez. Autrement dit, ce n'est pas toujours la lumière qui les y attirent. Les SHS ne peuvent pas montrer leur efficacité par le simple fait d'être applicables. L'enjeu de l'intervention dépasse très souvent l'aménagement. Elle concerne la transformation des organisations et cela ne peut pas se mesurer avec les instruments qui structurent le cadre qu'il s'agit précisément de changer. Et c'est alors un reproche qu'on peut bien leur adresser : les SHS ne pensent pas assez le changement de cadre comme un objet qu'il leur revient de traiter aussi. C'est donc le statut scientifique de l'intervention qui se trouve ici en jeu, sa valeur d'objet de recherche.

\section{Dispute sur l'intervention}

Pour de Montmollin, les faiblesses du taylorisme ne sont pas de principe ni de modèle, mais de développement. Aussi la vocation de l'ergonomie est-elle de compléter le taylorisme plutôt que le dépasser. Il s'agit finalement de l'améliorer, le moderniser (op. cit., pp. 19-20). Lors de la « Journée Christol » en prélude des Journées de Bordeaux 2003, il indique : «l'OST (l'organisation scientifique du travail) constitue l'horizon de l'ergonomie ». Cette phrase ne se comprend que si l'auteur raisonne dans le paradigme de l'ergonomie de la tâche, où l'amélioration des conditions de travail signifie l'aménagement plus scientifique des conditions de travail. Mais, M. de Montmollin s'inscrit résolument dans le paradigme de l'ergonomie de l'activité...

Le paradoxe peut être levé. En proposant de distinguer entre «ergonomie de l'utilisateur » et « ergonomie de l'utilisation », il fait bien apparaître dans l'ergonomie de l'activité de possibles « querelles d'écoles». Mais cette la frontière utilisation / utilisateur qui se situe tout entier à l'intérieur d'une conception fonctionnaliste de l'ergonomie, et donc ne suffit pas. Selon nous, il existe une autre frontière qui se franchit ou s'évite, semble-t-il, dans l'ergonomie de l'activité. À travers le fait de se servir de quelque chose - un produit, une machine -, il est en cause pour l'opérateur, et pas seulement pour l'objet, de servir à quelque chose. L'utilité est au fondement de la question du sens. Pour l'entreprise, c'est la vocation économique du travail ${ }^{3}$; et pour soi, c'est la vocation subjective du travail. Entre l'injonction cachée de l'entreprise que le travail comble les lacunes du sens et la vocation de l'homme de répondre par le travail aux sollicitations qui le touchent, il y a précisément ce que nous appelons le travail réel. Et toute la question «ergonomique » est de savoir si et comment ces appels se répondent, précisément... Et si cette question se pose, c'est que ce n'est jamais réglé d'avance...

Dans son ouvrage, de Montmollin ne raisonne jamais en termes de transformation des situations, donc jamais en termes d'intervention, mais toujours selon une définition de l'ergonomie qui n'exige d'elle que de fournir des connaissances applicables. La posture adoptée est d'expert. Ce n'est pas, selon nous, celle de l'ergonome. Si l'ergonomie s'en tient à fournir des connaissances applicables, alors son mode se limite à la recherche, et encore une certaine conception de celle-ci, celle qui se conclut dans des productions implémentables, agrégeables, et appropriables par des tiers. L'aide mémoire d'ergonomie pour la conception et la réception des postes de travail, conçu par Renault dans les années 80 , est à nos yeux un exemple réussi de fourniture et de transmission de connaissances applicables, que des utilisateurs ont pu s'approprier. Mais lorsque le cadre d'intervention n'est pas investi, l'ergonomie se contente de n'être qu'applicable. Cette ergonomie sans ergonome se résume vite au «bon sens », et favorise un consensus de façade. La reconnaissance commune d'un état donné passe pour une connaissance partagée du problème. Mais l'ambiguïté qui entoure alors le

3. Cette « définition » est en vérité insuffisante et doit être complétée : la question du sens pour l'entreprise doit être étendue à celle du sens de l'entreprise (pour la société) et donc la vocation économique de l'entreprise considérer dans un même mouvement la contribution de l'entreprise à la création de valeur, non seulement pour l'actionnaire, mais aussi pour la société. Cette dimension rejaillit en partie dans la question du sens pour soi, mais en partie seulement car la subjectivité ne se développe pas seulement dans le plan sociétal. Ce point est, pour les ergonomes, encore aveugle. Il faudra l'éclairer. 
statut des connaissances et du cadre d'application de ce document permet d' « humaniser » certaines contraintes, cela ne permet pas d'agir dans le contexte qui les produit, donc de transformer pour de vrai.

On se rappelle peut-être la formule de Hywel Murell : «the world needs ergonomics; it does not need ergonomists ${ }^{4}$... Notre position est exactement inverse. Ce dont le monde a besoin, c'est de personnes capables d'abord de transformer les situations de travail, pas seulement de connaissances qui sont nécessaires mais n'y suffisent pas et n'agissent jamais par elles-mêmes, par leur seule existence. Brutalement résumée, donc, notre position est que le métier d'ergonome, aujourd'hui, concentre beaucoup plus d'enjeux que l'ergonomie.

Nous espérons donc un meilleur horizon pour l'ergonomie que l'OST ou l'amélioration du taylorisme. Dans la transformation il est certes en jeu d'appliquer des connaissances mais aussi d'en produire d'autres. Cette production se réalise trop souvent dans la seule intimité des expériences d'ergonomes qu'un autre type de recherche, clinique, doit pouvoir constituer en savoir.

\section{Dispute sur l'organisation, et sur l'entreprise}

Telle que nous la comprenons, l'ergonomie de l'activité naît de la « découverte » que l'organisation ne peut pas être scientifique, parce qu'elle combine des logiques hétérogènes et hétéronomes. Par définition, il est impossible de parler de «solution» organisationnelle, il n'y a jamais que des « réponses ». Vue sous cet angle, la recherche ergonomique ne vise pas tant la conception d'outils nouveaux, mais la compréhension-promotion des conditions de leur usage. Identifier, comprendre, expliquer et tenir compte que ce qu'on conçoit ne « marche » jamais « comme ça »..., c'est le socle du savoir ergonomique.

Si on regarde cette question d'un peu plus haut encore, on retrouve la question du statut, du sens, de l'écart Tâche-Activité. Et découvrir qu'il faut distinguer encore entre des tenants qui pouvaient se croire d'accord. Quand M. de Montmollin fustige « une déviation sectaire minoritaire issue des discours humanistes d'Après dîner : la béatification de l'activité des opérateurs » (op. cit., p. 114), soit il raisonne comme un tenant de l'ergonomie de la tâche - ce qu'il se défend d'être -, soit il s'en prend à une conception de l'activité qui n'est pas la nôtre. Peut-être existe-t-il en effet des ergonomes assez « sectaires » pour considérer que le travail réel est nécessairement supérieur au travail prescrit, et pour soutenir en conséquence la supériorité de l'opérateur sur l'ingénieur. Mais là n'est pas pour nous l'enjeu principal de sa remarque. Ce n'est pas parce que l'opérateur n'a pas nécessairement raison contre l'ingénieur qu'il n'a pas toujours des raisons pour agir comme il le fait. C'est là que se jouent le statut et le sens de l'analyse du travail : rendre intelligible ce à quoi l'opérateur s'affronte, à quoi il est confronté - les dimensions hétérogènes et hétéronomes de son travail -.

L'ergonomie relève de ce que Latour appelle un exercice de diplomatie: nous ne nous situons ni entre l'ingénieur et l'opérateur, ni contre l'un ou l'autre, ces postures d'intégration ou d'exclusion constituant deux formes d'homogénéisation, mais avec. C'est-à-dire en recherche des tensions, confrontations comme autant de ressources pour co-opérer, avec aussi tous les « risques » que l'ergonome doit prendre, pour les autres et pour lui-même, dans une pratique dont la dimension éthique (par exemple, « se contenter » d'améliorer le taylorisme ou de proposer des alternatives) ne peut pas être dissociée de la dimension technique.

M. de Montmollin ne réfère jamais au terrain concret de l'ergonomie, l'entreprise, ce qui fait problème pour elle, pour les cadres, les décideurs, les syndicalistes, de sorte qu'il ne situe jamais son propos dans la perspective de la place du travail dans les modèles d'efficacité, ni de la représentation managériale - et syndicale - de la santé... Or, à l'inverse, c'est là pour nous la responsabilité même

4. Cité in Waterson P. E. et Sell R., (à paraître) 
de l'ergonome, le fondement de sa légitimité et de sa pertinence d'intervenant, que de comprendre ces tensions comme autant de leviers...

\section{Sur la responsabilité de ce que l'ergonome produit dans l'entreprise}

Les dirigeants d'entreprise formulent des demandes aux ergonomes plutôt dans le champ de la santé, mais attendent et contrôlent les propositions (piste d'actions, prescriptions ...) plutôt dans le registre de l'efficacité. Ils attendent des «applications » qui améliorent la performance du modèle (du modèle taylorien si tel est le cas), sans le remettre en cause.

S'il faut bien $y$ répondre, l'ergonome doit aussi en répondre. Améliorer l'efficacité (il faut généralement entendre "productivité ») est la condition exigée pour engager le coût d'une intervention. Comment l'ergonome pourrait-il se soustraire, alors, à la question de savoir dans quel système de valorisation économique du travail ses recommandations ou prescriptions « ergonomiques » sont attendues ? Conçoit-il sa proposition d'intervention en intégrant cette question ? Ou bien convientil que cela dépasse son « cadre»?

Cette exigence d'utilité de l'ergonomie est le plus souvent implicite de la part des dirigeants d'entreprise, et c'est à l'ergonome de situer son point de vue en regard des différentes doctrines de valorisation économique du travail.

De tout cela, les TMS constituent un cas d'école. Une «épidémie de TMS » sanctionnée par un «surcoût » brutal des cotisations d'assurance maladie constitue souvent, pour une direction, une situation d'urgence pour « faire de la prévention ». La commande est de diminuer les TMS, et en conséquence de faire disparaître les effets coûteux induits (surcotisations, gestion des inaptitudes et restrictions médicales ...). Pour un peu, on pourrait croire que les TMS constituent les seuls coûts non maîtrisés dans l'entreprise, et que c'est donc à la marge qu'il faut aborder cette question.

Dans la commande, l'ergonome est ainsi invité à bien comprendre : "écoutez, tout va bien, mais les TMS perturbent l'équilibre; on compte donc sur vous pour trouver des solutions, mais attention à ne pas toucher au reste, à remettre en cause cet équilibre... ». L'ergonome est ainsi sollicité dans un cadre qui oppose la prévention à l'efficacité. Si ce cadre n'est pas discuté, si l'ergonome ne décide pas de le renverser, l'intervention ergonomique ne pourra pas sortir de l'aménagement de l'existant.

Un exemple bien connu des consultants concerne le cas des TMS sur les lignes de montage. Supposons la situation où des opérateurs tendent le bras plus qu'il ne le faut pour saisir les pièces à assembler. Le poste est aménagé en rapprochant les boîtiers (application ergonomique de bon sens). Les amplitudes gestuelles se situent désormais à l'intérieur des normes biomécaniques de confort. Mais les plaintes continuent et les TMS aussi. Il se trouve en effet que les modifications de poste ont permis aussi de gagner en temps d'opération (aller chercher moins loin les pièces fait gagner en vitesse d'opération élémentaire)... Et ce n'est pas tout, car en effet, rapprocher les boîtiers participe aussi d'une économie de l'espace. Cette économie, répétée sur plusieurs postes de la ligne, fait gagner quelques mètres carrés au sol, et encore du temps de cycle... et ainsi de suite.

Quelles sont alors les conséquences observables? Les zones d'atteintes sont plus courtes, mais les gestes sont devenus plus rapides. Ils sont également aussi plus « compactés » du fait du rapprochement des postes voisins. Les possibilités d'encours entre postes sont diminuées voire totalement annihilées, de sorte que la nouvelle situation débouche sur une densification de gestes et une plus grande dépendance organisationnelle. Mais le bureau des méthodes a pu, de la sorte, gagner quelques points de productivité qui lui étaient demandé par ailleurs.

S'il se cantonne à répondre dans le « cadre » imposé par la demande, l'ergonome se sentira forcément «emprunté » (dans les deux sens du terme, c'est-à-dire manquer d'aisance et de ne plus s'appartenir en propre). Et chacun de se sentir manipulé, piégé, et de ressasser un cas de conscience : « j'attache la plus haute importance à ce que les pistes d'actions ergonomiques permettent aux opérateurs de travailler sans atteindre à leur santé; je veille à ce que ces pistes d'action soient réalisables techniquement; mais les transformations réalisées dépassent le projet sur lequel j'ai travaillé, intègrent d'autres projets qui m'ont échappés ou que je ne me suis pas autorisé à regar- 
der... L'entreprise (le client - la direction - le CHSCT...) évalue-t-elle mon intervention finalement au regard des seuls gains de productivité qu'elle pense réaliser ? reconnaîtra-t-elle son erreur en s'apercevant que la santé et l'efficacité des «personnes » ne sont pas préservées ou servirai-je de bouc émissaire?»

De ce qui risque d'arriver (et qui arrive forcément dans la trajectoire de l'intervention), l'ergonome doit en répondre. D'autant qu'il dispose de suffisamment de connaissances pour le faire, que ses interventions ont précisément produites. Les TMS témoignent certes d'une rigidification articulaire attentatoire à la santé des personnes, mais aussi d'une rigidification organisationnelle qui menace l'efficacité de l'entreprise. Ils résultent de la réduction des possibilités d'agir dans des situations où la diminution des marges de manœuvres organisationnelles des personnes pousse à une rigidification des comportement et qui menace la souplesse recherchée par l'entreprise (Bourgeois et al., 2000).

Prenons l'exemple d'ouvrières d'assemblage dans une industrie de biens d'équipement. Tous les ingrédients sont réunis pour fabriquer des TMS et la Direction le sait. Le service des méthodes accorde beaucoup d'attention aux aménagements de «confort» (distance et accessibilité des boîtiers, hauteur des coudes, etc.), mais il est aussi intéressé par la chasse aux opérations dites « sans valeur ajoutée », autrement dit des gestes « inutiles ». Un cabinet d'organisation est sollicité, et il invite les opératrices à assister aux analyses des séquences vidéo les concernant. Une «tâche sans valeur ajoutée » est repérée par un ingénieur : l'opératrice en bout de ligne retire de la balance la boîte pesée puis la dépose sur une table pour y coller une étiquette et saisir le code barre. Il est donc décidé que l'opératrice effectuera, désormais, ces deux dernières opérations sur la balance. Le déplacement de la balance à la table est donc supprimé. Quelques temps après, le responsable qualité est très ennuyé. L'opération de pesée, qui est une opération supplémentaire récente, avait permis de réduire spectaculairement les défauts qualité (manque de composants dans la boîte). Mais depuis la suppression du déplacement, les défauts ont subitement augmenté. Le gain en performance n'est donc pas au rendez-vous. L'explication est arrivée bien plus tard, dans le cadre d'une intervention ergonomique. Les opératrices distinguent volontairement le lieu de pesée et le lieu de pose de l'étiquette et de saisie code barre parce que les cadences de la ligne et de l'impression des étiquettes ne sont pas les mêmes. Saisir le code barre sur la balance a pour conséquence de ralentir la cadence de la ligne, et pour éviter cela, l'opératrice a tendance à retirer la boîte plus tôt, pour libérer la balance. Mais, faute de table, elle porte la boîte sur elle. Dans la double tâche (gérer deux flux différents au même moment), des confusions sont alors possibles. Le déplacement de la balance à la table s'avère donc une opération à valeur ajoutée, conçue par les opératrices dans une logique d'économie et d'efficacité de leurs propres actions. La table, enjeu de possibilité d'agir pour les opératrices, a été réintroduite.

Dans notre exemple, le modèle d'efficacité qui inspire la suppression de la table de dépose est classique, taylorien. En cherchant une plus grande rentabilité par une réduction du coût des opérations de travail, le service méthodes a créé une densification du travail qui est devenue une source de non productivité et d'atteinte à la santé. Les ouvrières sur la ligne d'assemblage ne peuvent plus contribuer à la qualité alors qu'elles savent le faire, ni se protéger des risques musculo-squelletiques alors qu'elles savent aussi probablement le faire.

Les TMS sont bien, ici, un symptôme de rigidité dans une organisation qui visait la souplesse.

Cet exemple, et tant d'autres dans les entreprises, invite à questionner les modèles de valorisation économique du travail. L'enjeu est de traduire les connaissances de l'activité du travail non pas seulement dans le champ de la transformation technique mais aussi en alternatives organisationnelles. Dans notre exemple, le retour de la table de dépose ne constitue certes qu'un aménagement du modèle taylorien, mais il montre l'inadéquation de ses fondements au regard des conditions dans laquelle l'activité se réalise. Dans un contexte ouvert à la conception des systèmes de travail et de production, il légitime des alternatives opérationnelles. C'est, en tout cas, de cette façon que l'approche ergonomique est reçue par les organisateurs, comme une nouvelle possibilité de penser les modèles d'efficacité. Ce que coûte la réintroduction de la table et les gestes " chassés » vaut, bien sûr, ce que coûte la non qualité sans cette option. L'objectif de productivité de l'entreprise est le même. Mais les moyens pour y parvenir et les résultats sont différents. 
Les entreprises n'ignorent pas ces contradictions. Mais, sans prétendre à l'universalité de l'ergonomie, elles ne peuvent les travailler que si nous nous y engageons aussi. Les personnes des méthodes, par exemple, évoquent leur malaise à trop «tirer sur la corde». Faire de la prévention des TMS, c'est pouvoir et savoir instruire la critique de certaines formes d'organisation et de management, voire des modèles de gestion. C'est, aussi, mettre en perspective des alternatives. En d'autres termes, les prescriptions ergonomiques valent en fonction de ce qu'elles ouvrent comme perspectives à la crise des modèles d'efficacité auxquelles sont confrontées les entreprises.

\section{En guise de conclusion : disputons-nous !}

Emblématique d'un human engineering dont pourtant il s'attache à se démarquer, le raisonnement de M. de Montmollin se déploie hors d'atteinte - et donc de réponse - des questions d'efficacité (qualité, fiabilité, ...) ou de santé (stress, épuisement, TMS, ...) ou de développement opérationnel (culture de sécurité, de sûreté, démarche client, sens du service public), et a fortiori des liens - or c'est là l'enjeu même de l'organisation - entre ces questions. C'est que ces questions-là, les modèles fonctionnalistes ne peuvent ni ne cherchent à en rendre compte. Ces rapports relèvent des dimensions de l'activité (implication, compréhension, décision, engagement...) qui ne sont précisément pas fonctionnelles.

Les écoles se distinguent par leurs doctrines, c'est-à-dire les corpus des croyances partagées par les praticiens et les chercheurs qui se réclament de la même école. Mais à l'intérieur de la même école " peuvent se dégager des courants qui, par leurs élaborations théoriques et scientifiques, infléchissent la signification, voire la formulation de la doctrine. Actuellement on peut considérer que l'ergonomie dite de langue française constitue une école, dont la doctrine sur les relations entre l'homme, la santé, la technique, le travail et les valeurs se distinguent assez explicitement de la doctrine des "human factors" et de l'ergonomie anglophone» (Dejours, Op. cit., p. 215).

Avec la position soutenue par Maurice de Montmollin, est-ce affaire de courant ou d'école ? On accordera que la question ici n'a d'intérêt que dans la perspective de travailler ces différences, ce qui suppose de les comprendre, et de refuser tout autant de les caricaturer comme de les taire, sauf à ce que l'ergonomie de langue française, et incidemment la Société chargée de l'animer, ne discute plus de rien sur le fond. Un point de vue n'existe que parce qu'il y en a d'autres, et il approfondit sa singularité en la confrontant à d'autres. L'absence de controverse agit comme l'hypnose : envisager indéfiniment le même objet du même point de vue, et il devient impossible de le voir... La manière d'être ensemble est donc en cause si on ne débat plus.

\section{2.- Une ergonomie « centrée » sur l'activité}

\section{L'activité témoigne de l'inaliénabilité du travail}

Pour nous, c'est bien l'inaliénabilité du travail (i.e. sa vocation à l'autonomie) qui permet que l'Homme trouve les moyens de compromis opératoires [i.e. d'une activité "discrétionnaire » (Maggi, 1996)] dans des situations aliénantes (i.e. hétéronomes).

En nous inscrivant dans cette visée, c'est le mode par lequel l'homme participe à l'élaboration du sens qui fait problème, autrement dit l'expérience par laquelle ce qui arrive lui arrive effectivement ${ }^{5}$. Pour comprendre ce qui nous arrive, il nous faut bien être pris dans l'histoire qui se joue, et nous

5. Pour Baudrillard (1997, p. 128), « le réel est ce à quoi il ne faut pas consentir : il nous arrive alors que nous devrions lui arriver... le réel n'est jamais qu'un modèle de simulation et de réglementation du devenir... un modèle de réduction de la singularité » (p. 129). 
sentir concernés par les événements. Ainsi, l'enjeu de toute activité c'est de résister à son aliénation, et faire advenir une histoire qui ne nous parvient le plus souvent que par bribes.

Travailler, c'est s'opposer. C'est là le sens que nous donnons à la formule de Cazamian: « l'ergonomie est la science du travail aliéné ». Elle témoigne de l'élaboration d'une pensée dans le travail et par le travail, et par là même de son inaliénabilité.

Reconnaitre l'inaliénabilité du travail, c'est l'impliquer dans une histoire qui le modifie, c'est le rendre disponible pour une autre histoire que celle où l'analyse le révèle. L'activité ne se limite jamais au réalisé ; le voulu, le possible et l'impossible font tout autant partie du réel du travail. Dès lors, être reconnu dans ce qu'on fait, c'est aussi être reconnu pour ce qu'on peut et veut devenir d'autre. Comme le dit Clot, l'autonomie c'est ne pas dépendre de soi comme d'un autre, et il nous semble bien que l'inaliénabilité du travail se joue là : dans l'usage que l'on fait, dans le travail, de l'hétéronomie.

\section{Un exemple :}

La valorisation de l'investissement subjectif dans le travail pose un vrai problème à l'ergonomie. Comment tenir ensemble l'objectivation de données « dures » (limites de sollicitations musculaires, processus de discrimination des signaux ...) et la prise en compte de réalités pudiquement présentées comme « subjectives»?

Une leçon magistrale a été donnée par des peintres de boucliers de voiture à qui une équipe d'ergonomes restituait ses observations sur des postures « pénibles » (au regard des connaissances en physiologie et biomécanique, elles devaient être " pénibles »). Les peintres ont fait comprendre qu'ils ne ressentaient pas cette pénibilité, mais plutôt du plaisir à faire de la sorte : «il faut nous voir danser ! ». Ils ont fait comprendre aussi qu'à vouloir s'en mêler comme ça, les ergonomes risquaient, cette fois, de leur rendre pénible la vie au travail.

Que signifie cette incursion de l'opérateur dans l'espace méthodologique de l'ergonome? Que la valeur limite du plaisir au travail ne se décrète pas de la même façon que l'accélération du rythme cardiaque dans un local chauffé à $25^{\circ} \mathrm{C}$, lors du portage d'un carton de $20 \mathrm{~kg}$, sur un plan incliné de $10^{\circ}$. Bien sûr, on peut «neutraliser» cette variable, autrement dit on peut ne rien entendre, rester hors d'atteinte.

L'équipe d'ergonomes a cherché plutôt à dépasser son embarras et à comprendre ce que ces postures pouvaient «fabriquer » d'autre que la pénibilité à laquelle les « données » concluaient normalement. Pour maintenir une distance et une vitesse d'application de la peinture les plus constantes possibles, les peintres s'engagent effectivement très physiquement d'un bout à l'autre du bouclier. Mais ils règlent leur vitesse et leur distance en évaluant, à partir d'informations visuelles, la densité de peinture déposée. Les postures « dites pénibles » sont donc justifiées par la nécessité de faire des passes complètes, de prendre des informations rapidement et de corriger éventuellement par des passes supplémentaires là où la peinture fait défaut. Bref, « ils fabriquent de la qualité », au moyen de régulations (de règles « chorégraphiques » pourrait-on dire) construites collectivement. Et cette qualité s'appelle « homogénéité de la peinture sur le bouclier ». Pour les responsables de l'entreprise questionnés sur ce critère, l'homogénéité n'est pas un problème. L'homogénéité est pour eux normale, quasiment naturelle, comme si elle procédait d'un phénomène de génération spontanée. Leur problème de qualité, à eux, c'est la « présence de grains sur le bouclier », attribuée aux déplacements et aux postures en tous sens des peintres qui brassent de la poussière...

Avec cet exemple, nous sommes confrontés à deux modèles de contrôle de gestion de la qualité.

- L'un, officiel, compte les grains et conclut que les modes opératoires posturaux des peintres ne sont pas efficaces puisque les grains sont identifiés à l'origine.

— L'autre, objectivé à la suite d'une interpellation d'un opérateur à des ergonomes, « compte » les déplacements comme une ressource qui permet d'empêcher les défauts d'homogénéité et conclut que les mêmes modes opératoires posturaux des peintres sont, là, efficaces. 
Finalement, les ergonomes ont contribué à la compréhension du modèle opératoire des peintres qui a gagné le statut d'efficacité aux yeux des responsables de l'entreprise. Au lieu de prescrire l'interdiction de bouger, la possibilité de déplacement de part et d'autre du bouclier a été maintenue.

Pour avancer, l'ergonome a été obligé de questionner les instruments de mesures de l'efficacité de l'entreprise, et de fait, le modèle de contrôle de gestion. Une mauvaise représentation des conditions dans laquelle l'efficacité s'obtient a des effets immédiats sur la façon dont l'entreprise pilote ses projets et son organisation. Le diagnostic de départ étant mauvais (ici l'ignorance des décideurs de la façon dont se fabrique l'absence de défauts d'homogénéité), les prescriptions l'étaient aussi (interdiction des déplacements...). Si on laissait en l'état, les peintres voyaient les possibilités de maîtrise de la qualité se réduire et leur latitude pour gérer leur santé (ne plus prendre plaisir à engager son corps pour faire du beau travail...) aussi. C'est bien la proposition d'un autre système d'informations et d'évaluation de son efficacité qui a incité l'entreprise à redéfinir son projet.

Ce que coûte, ici, le réinvestissement dans les déplacements des peintres et leur « liberté d'action » vaut, bien sûr, ce qu'auraient coûté les défauts d'homogénéité s'ils ne pouvaient plus se déplacer. Dire cela, c'est rappeler que le travail a certes un coût mais aussi un effet utile, et qu'il est à ce titre un vecteur de réduction des coûts, une source d'efficacité, tant économique que subjective, on le voit.

L'analyse de l'activité ne peut pas ignorer la subjectivité du travailleur, ni se passer de se confronter aux modèles d'efficacité de l'entreprise. Observer, comprendre et expliquer le rapport entre santé et efficacité, c'est la responsabilité et l'objectif stratégique de l'intervention.

\section{Activité, un concept trop large pour l'ergonomie ?}

Pour nous, l'ergonomie a en charge de permettre qu'autre chose advienne du travail. L'analyse du travail, ainsi, a en charge le développement du travail, c'est-à-dire l'approfondissement de sa singularité.

L'homme pense parce qu'il a des mains disait déjà Anaxagore. De fait, c'est par l'exercice d'une intelligence fabricatoire que, en manipulant le monde, il l'apprend et le comprend. Le corps n'est pas chose mais accès aux choses. Système de pensée et système corporel ne peuvent être isolés et « le sentir et la pensée ne se laissent pas séparer » (Böhle \& Milkau, 1998). Ensemble, ils forment un processus, à penser lui-même. Faire corps avec son appareil indique assez comment la motricité organise la pensée du pilote. Donner corps à un projet, indique aussi que rien n'existe tant qu'on s'en tient à l'énoncé, et qu'il faut bien qu'à un moment, les choses s'incarnent, s'éprouvent...

Ainsi, toujours, l'acte est une aventure (Mendel, 1998) : c'est dans l'acte du travail qu'on prend acte du monde, et c'est parce que cette expérience est pleine de surprises, et d'étonnement, que, tout n'étant pas prévisible à l'avance, il y a engagement et qu'il y faut mobiliser de la ruse et de la prudence.

La pensée occidentale nous a pourtant incités à une toute autre approche. Elle a plutôt consacré un face à face du corps et de l'esprit, au point, selon Mendel, que l' " acte » y reste littéralement impensé. Absorbé dans l'action (l'idée, l'intention, le projet), l'acte est considéré comme un simple ou vulgaire reflet du projeté et ne mérite pas qu'on s'y attarde. L'acte normal est censé n'être que l'actualisation de la puissance de l'action, l'exécution du programme, ou du projet. La danse des peintres dans notre illustration précédente, est un " acte » nécessairement inintelligible dans un modèle taylorien de l'efficacité, et de ce fait nécessairement un défaut.

Mais, entre l'action et l'acte, Mendel fait-il voir la même coupure que celle que traditionnellement les ergonomes reconnaissent entre travail prescrit et travail réel ? L'action, dit-il (p. 8), c'est « ce qui se passe "dans la tête"... quant à l'acte, il concerne (...) l'interactivité du sujet et d'une réalité qui lui est étrangère - autrui, la société, la nature ». Ainsi, « il n'y a plus véritablement d'acte dans le "travail en miettes" dans la mesure où il n'existe plus un sujet» (p. 46, note 11). 
Par rapport à la tradition ergonomique, la distinction n'est donc pas exactement la même : Tâche et Activité renvoient, à titre principal, à une répartition sociale et organisationnelle des rôles tenus par des acteurs différents - le rôle de conception des «ingénieurs» et le rôle de réalisation des « travailleurs »-, quand Action et Acte expriment plutôt les termes du débat intime de celui qui agit, débat qui est généralement « impensé » par celui qui programme et complètement ignoré des modèles qui en font l'analyse. Mais si on fonde la différence Tâche / Activité sur l'hétéronomie et l'hétérogénéité, alors la coupure résonne en symbiose.

Il nous semble que la même tension règle le couple style /genre analysé par Clot. Au delà (ou en deçà) des règles qui s'imposent à eux, les travailleurs s'imposent, eux-mêmes, à eux-mêmes, des formes prescriptives, ne serait-ce que pour économiser leur effort. Le genre, ainsi ${ }^{6}$ cristallise sous formes de «manières de faire » à la fois les « composantes impersonnelles de l'activité subjective » que forme l'ensemble de ce qu'on sait devoir faire, les « obligations du métier » (Clot, 2000; Clot \& Faïta, 2000), mais aussi tout ce que l'expérience de chaque sujet programme en lui - Clot (2000, p. 279) parle à ce propos de « genre intérieur »-. On voit bien la résonance du genre avec la tâche, surtout après que Clot lui-même a entrepris de définir la tâche comme « le modèle refroidi » de l'activité des concepteurs (Clot, 1995, p. 212). On perçoit bien ici pourquoi et comment Clot vise à renouveler l'analyse francophone de l'activité. Au sens «classique », la «tâche » reste l'épreuve d'un autre que celui qui réalisera le «travail réel ». Il faut lui ajouter le poids des exigences que le sujet hérite de sa propre histoire. Et c'est pourquoi alors, à son tour, le style est une notion plus riche, selon Clot, que l' " activité » : « le style est ce troisième terme qui vit aux confins des conflits qui agitent les deux mémoires de l'activité ${ }^{7}$....(c'est) un "mixte" qui signe l'affranchissement possible de la personne vis-à-vis de sa mémoire singulière, dont elle reste pourtant le sujet, et de sa mémoire impersonnelle et sociale dont elle reste forcément l'agent »(Clot, 2000, p. 279).

Si le style complète en permanence le genre, c'est, selon Clot, parce que le genre est toujours inachevé. L'ergonome dira plutôt que si l'activité retravaille la tâche, c'est parce que la tâche est incomplète, en partie du fait même qu'elle est extérieure.

Dans un registre un peu différent, enfin, le couple Conformité/Originalité, qui soutient le « jugement de beauté » (Dejours, 1995, p. 61-62), offre un autre écho. Entre le genre et la conformité n'y a-t-il pas forte résonance en effet, et alors avec l' " action » peut-être, et la tâche aussi, avec toutes les réserves que nous venons de faire ? De même, le style et l'originalité, n'ont-ils pas partie liée avec l'activité, et celle-ci avec l' '" acte »?

Bien sûr, la perspective théorique et pratique n'est pas la même. Dejours s'intéresse à la reconnaissance de la compétence de l'individu par ses pairs qui jugent tout à la fois la conformité de sa manière de faire avec les règles de l'art en vigueur, et sa capacité à déborder ces règles pour les mettre à l'échelle du cas particulier qui les sollicite.

La question soulevée par Dejours ne concerne donc pas le mode de constitution de la conformité et de l'originalité, mais plutôt sa fonction subjectivante ${ }^{8}$. Il n'empêche, Dejours souligne aussi le rôle du jugement d'utilité dans la reconnaissance de la compétence, et la question est bien alors de comprendre comment ces deux jugements se croisent. Tout cela ne fait-il pas alors écho avec le fait que la question soulevée par Clot concerne aussi la dimension subjectivante du genre et du style - ne serait-ce qu'à travers le rôle du sujet, et du groupe, dans la «mise en patrimoine »-, et avec la manière dont l'ergonomie comprend le travail comme gestion du lien entre santé et efficacité ?

6. Du moins est-ce là l'interprétation que nous faisons ici de ses propos...

7. La mémoire « impersonnelle et sociale » des devoirs de la tâche, et la mémoire subjective de l'expérience du sujet.

8. Pour être exact, Dejours s'intéresse ici au jugement porté par des tiers, et non à la manière dont l'individu travaille réellement en conformité et en originalité. 


\section{En résumé,}

- Pour Mendel, la prescription est une catégorie essentiellement épistémologique, qui non seulement exclut toute possibilité de penser le travail comme un «acte », mais menace l'acte luimême de ne plus savoir tenter l'aventure, et de se laisser enfermer dans l' " action », de faire trop crédit au programme et perdre ainsi toute puissance réelle.

- Pour Clot (et Dejours dans la référence limitée que nous venons d'y faire ici), la prescription est à la fois un processus social hétéronome et un processus collectif autonome.

- Pour l'ergonomie "classique», la prescription renvoie à un usage de soi par les autres (Schwartz, 2000), elle sanctionne une distance tout à la fois épistémologique, sociale et politique dont le couple concepteur / exécutant est la forme achevée. En disant « classique », nous ne prétendons pas diminuer cette position. Elle reste la notre. Nous voulons seulement dire qu'elle doit être approfondie du fait que l'ergonome doit prendre acte qu'il y a plusieurs prescripteurs dans la prescription, y compris l'opérateur - sujet individuel et collectif - qui cherche dans son activité, non seulement à corriger les insuffisances de la tâche face à la singularité des situations réelles, mais aussi à faire reconnaître ses propres exigences singulières, ses attentes... les prescriptions d'un usage de soi par soi..., attestant ainsi de l'inaliénabilité de l'autonomie dans les activités hétéronomes.

L'ergonomie a réglé (?) en son temps sa frontière entre l'individu et le « collectif» (rappelons-nous les débats sur la frontière entre l'ergonomie et l'anthropo-technologie proposée par Wisner comme « solution » à cet élargissement de l'horizon théorique et politique de l'ergonomie). C'est celle du «sujet» qui fait aujourd'hui son actualité théorique. Quels rapports saurons-nous établir avec la psychologie clinique et la psychanalyse?

\section{3.- Le singulier est une synthèse}

Pour développer une approche globale du local, la démarche clinique s'appuie sur un dispositif permettant de comprendre ce qu'elle fait. Un dispositif, c'est-à-dire à la fois un cadre (un agencement structuré) et un processus.

L'intervention est toujours confrontée à une forme particulière de la fameuse «guillotine de Hume » : on ne peut agir sur la réalité qu'en empruntant le détour dialectique d'une valeur hiérarchiquement supérieure. Cela signifie, pour nous, que le changement des situations de travail passe par le détour, non seulement des valeurs qui fondent ce que les personnes attendent de leur propre activité, mais aussi des valeurs qui structurent, à travers ce que l'entreprise attend du travail, ce qu'elle demande aux personnes qu'elle sollicite. Il nous semble, ce disant, que toute démarche d'intervention participe de ce qu'on appellera un optimisme méthodologique. Cela suppose en effet de croire à la nécessité et à la possibilité de ce détour, à condition, bien sûr, qu'on sache penser l'intervention en termes de dispositif, épistémologique, méthodologique et politique, renouvelé. C'est en cela que l'intervention constitue un objet de recherche, central pour l'avenir de l'ergonomie comme métier.

\section{Dispute sur la scientificité dans et de l'intervention}

L'activité de travail n'est pas réductible à ce qui s'objective dans le faire. A fortiori, aucune description n'est à même d'épuiser la pensée qui se réalise dans le travail. C'est par le concept d'activité que l'ergonomie ambitionne de penser le travail comme une pensée, et rendre publiques les dimensions cachées dans ce qu'on voit du travail, ou ce qui s'en dit.

Il s'agit de rendre compte d'un double mouvement : celui qui donne au travail son pouvoir de transformer le monde, le pouvoir de l'acte - i.e. la question de l'efficacité du travail -, et celui qui donne au sujet le pouvoir dans son acte - i.e. la question de la subjectivation par le travail -. Comprendre la 
relation entre ces deux mouvements, c'est la vocation de l'ergonomie, qui doit pour cela faire écho à la manière dont l'« opérateur » pense lui-même cette relation dans le mouvement même qui la réalise.

En termes épistémologiques, il est en effet nécessaire que l'unité de base de l'analyse contienne les propriétés fondamentales de ce qu'on cherche à analyser. L'ergonomie ne pourra jamais rendre compte du travail comme activité de pensée, si elle ne se constitue pas elle-même comme dispositif de pensée sur le travail et donc si elle n'ambitionne pas des systèmes de travail alternatifs.

L'enjeu de la démarche ergonomique est de connaître, reconnaître, pour transformer. Au plan de l'analyse de l'activité de travail des « opérateurs », c'est l'enjeu de la reconnaissance de leur compétence, de la préservation des atteintes à leur santé ; au plan de l'intervention ergonomique (l'activité de travail des ergonomes). C'est aussi l'enjeu du type d'espace politique ouvert par l'intervention. Ce n'est possible que dans le cadre d'une ergonomie de l'activité qui n'aborde pas la tâche de la même manière que celle proposée par l'ergonomie de la tâche... On est donc là au cœur de ce qui se joue dans l'ingénierie de projet : faire de la tâche le moyen de l'activité et non pas son horizon. Cela passe par une définition de la tâche non pas comme norme, mais comme référence.

Chacun sait que toute observation prolongée agit et construit donc un objet qui devient, ainsi, le résultat de ce que l'observation produit... La question est de comprendre comment on peut se servir de cette dynamique pour penser l'acte de transformation de la réalité qu'on "observe ». Pour Girin (1990), c'est l'intérêt de penser l'intervention en termes de rationalité interactive, et pour cela de la concevoir comme un dispositif de recherche.

Pour lui, il y a deux manières de poser la question du travail scientifique :

- celle qui s'intéresse à «la science telle qu'elle se fait» comme dit Latour (1989), et qui s'inquiète de comprendre comment les scientifiques travaillent;

- et l'autre, purement normative, qui s'inquiète de ce que les scientifiques devraient faire pour mériter ce titre.

Pourtant, il n'y a pas vraiment deux manières de prétendre à la scientificité. Comparer sa pratique à celle de ceux dont la scientificité est reconnue, ce n'est pas vraiment probant : la diversité des pratiques est infinie, la comparaison difficile, et seul compte alors de vérifier si «ça a marché ». Reste donc la perspective normative.

Popper (1934) est certainement la figure emblématique de cette perspective. L'exigence poppérienne est en effet parmi les plus contraignantes, surtout dans les organisations sociales où la réalisation du test de la réfutabilité est particulièrement problématique. Mais Girin montre que la thèse de Popper est moins rigide qu'on ne le dit. L'idée centrale est que « l'objectivité de la science n'est pas une question d'individu, intéressant les hommes de sciences pris à part, mais une question sociale qui résulte de leur critique mutuelle, de la division du travail amical-hostile entre scientifiques, de leur collaboration autant que de leur rivalité. Elle dépend donc partiellement d'une série de conditions sociales et politiques qui rendent cette critique possible $»^{9}$. Indiscutablement, ce commentaire « humanise » la rigueur de l'exigence poppérienne. Et, par ailleurs, il ne met pas les sciences humaines et sociales en plus mauvaise posture que les sciences renommées plus dures. Sauf pour ceci, tout de même : Girin souligne que nos démarches relèvent de l'opportunisme méthodique, l'activité de l'intervenant n'étant que rarement conforme au plan prévu, du fait même qu'il s'adapte au terrain et cherche à épouser les lignes de forces susceptibles de favoriser son projet.

9. In (Girin, 1990, pp. 173-174) 
Qu'en est-il alors de la prétention à la scientificité de l'intervention - et nous rejoignons ici la dispute engagée plus haut avec M. de Montmollin - ? Selon Girin, Popper propose deux sortes de réponses :

- être fidèle aux faits, c'est d'abord rendre compte de «l'histoire qui nous intéresse » à travers eux ${ }^{10}$. Les sciences sociales n'ont pas uniquement pour vocation d'établir des lois universelles. Elles ont aussi à établir et expliquer des faits particuliers qui répondent à des lois qui ne sont pas nécessairement à découvrir. La valeur d'un fait ne dépend pas seulement de sa généralisation, elle répond aussi de l'intention poursuivie dans sa « découverte ». Pour nous, cette intention concerne la tension entre l'efficacité qui renvoie aux attentes de l'entreprise, ce qu'elle demande à travers la tâche et, d'autre part, la santé où il s'agit de considérer les exigences de construction et de développement de la personne, qui renvoie à ses propres attentes, ce qu'elle vise à travers son engagement dans l'activité.

- Popper distingue deux sortes de prédictions scientifiques : la prédiction historique (ex : un typhon va survenir) et la prédiction technologique (ex : l'abri va résister); il préconise que les sciences sociales s'intéressent aux « logiques de situation » et se livrent à des «manipulations » locales plutôt qu'à des prophéties, et affinent ainsi leur efficacité « sociale fragmentaire ».

Le concept de réfutabilité diversifiée que nous avons proposé (Hubault, 1996) est évidemment de résonance poppérienne ${ }^{11}$. Nous soutenons que l'intervention ergonomique, comme Girin le revendique pour la gestion, est une pratique de recherche dès lors qu'elle ambitionne un certain degré de généralisation à partir des faits particuliers sur lesquels elle peut établir son influence ${ }^{12}$.

Pour autant, le débat épistémologique ne se clôt pas avec Popper, et Girin a raison de faire valoir qu'il laisse entièrement sous silence la question de la compréhension, c'est-à-dire de la valeur de la « manipulation » pour ceux qu'on « étudie ». L'intersubjectivité est au cœur de l'intervention, et elle participe d'un processus qui relève de l'analyse du processus de transformation engagé. Sa valeur épistémologique ne saurait être sous-estimée.

\section{La multidisciplinarité comme dispositif d'intervention}

L'enjeu de la construction multidisciplinaire, ce n'est pas d'opposer une analyse à une autre, mais de changer, par leur confrontation, le cadre de l'analyse, et donc de l'intervention. La multidisciplinarité en ergonomie vise donc un changement de cadre, de l'analyse et de l'intervention sur le travail, et c'est ce changement de cadre qui, littéralement, la sépare des disciplines qu'elle sollicite. C'est dans cette divergence que se juge sa pertinence épistémologique et politique. Faire de l'ergonomie, c'est décider de la manière de faire diverger les « sciences mères » qu'elle assemble, vers un objet commun nouveau.

Si on monte d'un cran dans l'intervention, l'ergonome a à construire un cadre multidisciplinaire de maille plus large avec ses partenaires dans l'intervention. Construire l'objet d'une intervention multidisciplinaire s'avère une question sensible qui sollicite en effet l'accord, non seulement des « clients », mais aussi des partenaires dans le collectif d'action qu'elle amène à constituer. Tomber d'accord définit ici aussi une condition fondamentale de la coopération, mais tomber d'accord sur les raisons et la manière de diverger ensemble, rien de moins évident!

10. In (Girin, 1990, p. 176)

11. Voir infra, les conséquences de cette assertion, en termes de dispositifs d'évaluation à concevoir, pour soutenir la pratique de l'ergonomie.

12. Il nous semble que Daniellou $(1996,1997)$ fait à peu près le même pari, en soutenant que « l'ergonomie intervient dans et sur des situations de gestion », concept développé par Girin (1990, p. 142) : " une situation de gestion se présente lorsque des participants sont réunis et doivent accomplir, dans un temps déterminé, une action collective conduisant à un résultat soumis à un jugement externe ». 
Trois logiques s'emmêlent toujours dans toute intervention, qu'il faut retrouver pour juger de sa valeur :

- la logique des domaines dans lesquels l'ergonomie choisit de s'investir ${ }^{13}$;

- la logique de la discipline ergonomique ;

- la logique du projet.

A partir de là, toute intervention se déployant dans un univers d'événements qui requièrent de mobiliser un système de valeurs pour y faire face ensemble, agir ensemble, exige de partager un certain nombre de principes et d'intentions, et la capacité de les retraiter ensemble au fur et à mesure qu'ils seront mis à l'épreuve des événements rencontrés.

La forme la plus modeste de la scientificité consiste donc à tenir que rester fidèle aux faits, c'est rester fidèle à " l'histoire qui nous intéresse » à travers eux. Précisons bien que nous entendons par-là, tout à la fois que les « faits » nous intéressent par rapport à la visée qui les saisit (ce que Bakhtine, appelle « le paradigme extopique »), mais qu'il ne s'agit pas pour autant de ne retenir pour probant que ce qui conforterait cette visée ${ }^{14}$.

Cette fidélité positionne l'ergonome.

Positionnement doit s'entendre tout autant dans une dimension conceptuelle que sociale. Cela relève du mode de présence de l'intervenant dans la question qu'il traite, tout autant méthodologiquement que socialement, ou politiquement. Intervenir dans une perspective clinique, c'est intervenir d'une manière où l'instrumentation technique et l'instrumentation sociale sont, au final, pratiquement et épistémologiquement liées ${ }^{15}$.

Une conclusion s'impose alors : tomber d'accord, enjeu de toute coopération dans l'intervention, peut aussi bien se dire : qu(i)'est-ce qu'on laisse de coté ? Cela renvoie :

- à la responsabilité de l'intervenant (enjeu éthique) ;

- à la compréhension de l'intervenant (enjeu d'intelligibilité).

\section{Pour une conception clinicienne de l'" analyse de l'activité »}

Bien plus qu'une méthode et une technique, la démarche clinique est d'abord un positionnement général, par rapport aux autres et par rapport au savoir et à son élaboration : « elle comprend deux faces complémentaires : l'acte lui-même d'intervenir, c'est-à-dire une forme particulière de répondre à une demande, et une pratique de recherche d'autre part, directement impliquée dans les processus de changement ainsi engagés et rompant avec les principes du positivisme scientifique » (Lévy, 1997, p. 14).

Ainsi trois dimensions sont à relier. Suivons ici Lévy (Figure 1). Il préconise un dispositif en «trois moments » (p. 89) qui ne prennent sens qu'ensemble :

- l'acte, ou le travail clinique proprement dit, qui met en rapport l'intervenant et les sujets porteurs de la demande d'intervention ;

- l'élaboration théorique, où les intervenants reprennent l'intervention après coup avec d'autres cliniciens intéressés à formaliser la compréhension de cette reprise;

13. Par exemple, nous défendons l'idée que la gestion constitue un « domaine » d'intervention, et non pas seulement un contexte (Blazejewski et Hubault, 1999)

14. Tout le problème de l'exactitude des sciences humaines tient alors à la difficulté de « surmonter ce qui est autre sans le transformer en quelque chose à soi » « (Bakhtine, in Clot, 1995, pp. 176-177)

15. Sur cette question, se référer à l'ouvrage collectif édité par Daniellou (1996). 
- la communication de ces acquis, souvent sous forme écrite, à un ensemble plus large que la communauté impliquée dans l'acte clinique.

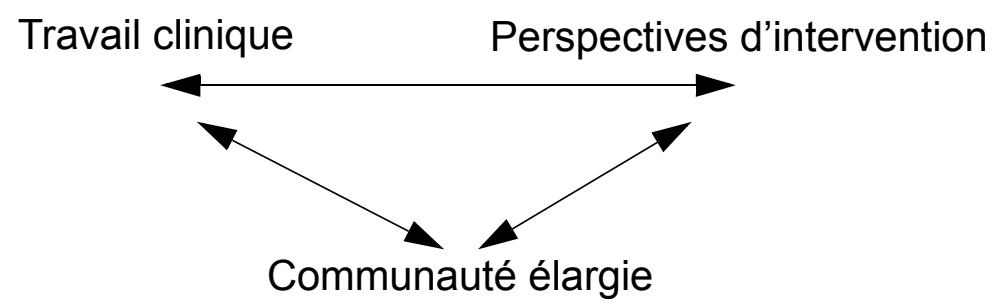

Figure 1.- Le dispositif en « trois moments » (d'après Lévy, 1997)

Schwartz (1996) propose un dispositif un peu différent (Figure 2). Il préconise quant à lui, un « dispositif à trois pôles » (p. 156), très proche dans l'esprit de celui de Lévy, plus complet selon nous dans le commentaire qui le soutient. L'idée centrale est que l'intervention ne peut pas être regardée à la seule échelle des intervenants présents dans l'action, et que l'intervenant ne peut revendiquer aucune « exterritorialité » :

- ni envers la situation d'intervention, puisque le rapport entre l'activité des opérateurs et les valeurs qu'ils y engagent est constamment retraité dans leur activité-même et ne peut donc jamais être objectivé a priori, ni s'objectiver hors de la présence agissante de l'intervenant ;

- ni envers son propre système de valeur qui supportera les mêmes oscillations au cours de son activité dans l'intervention.

Les choix conceptuels, les « bifurcations » (ces divergences que l'intervention occasionne) ne pouvant donc pas être faits " en totale neutralité... », il est essentiel que l'intervention s'inscrive dans un réseau où le "rapport triangulaire entre valeurs, activités et savoirs devienne l'objet d'une épistémologie explicite ».

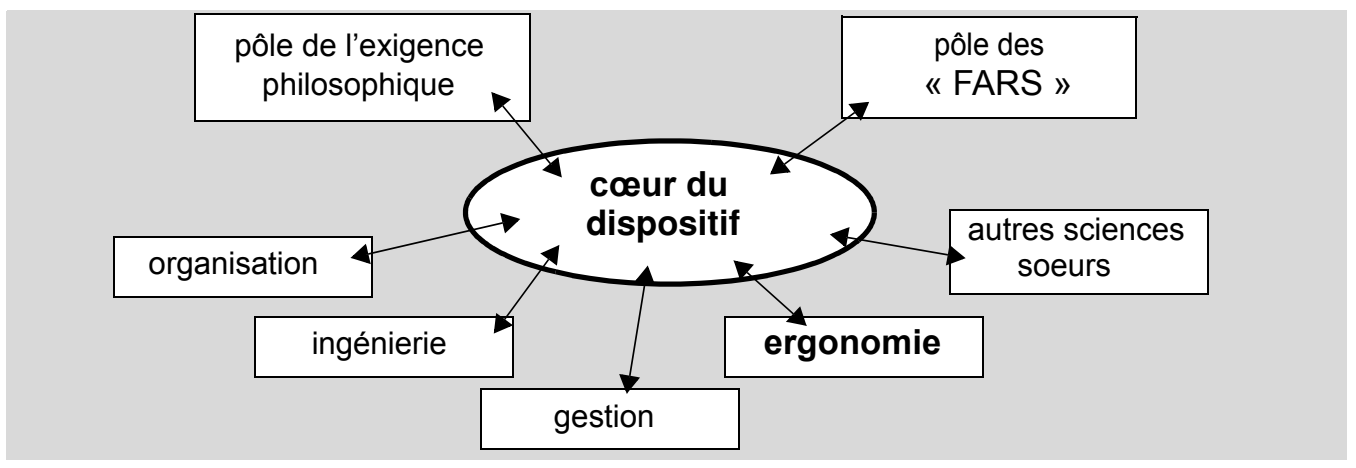

Figure 2.- Le « dispositif à trois pôles » (Schwartz, 1996).

Chacun de ces deux dispositifs, avec sa manière propre, répond à deux préoccupations liées : jouer conjointement le coup et la partie, et gérer les tensions internes entre logique métier et logique projet. Ce dispositif se structure par ce que nous avons appelé le principe de réfutation diversifiée, qu'il décline sous deux faces : 


\section{Épistémologique :}

L'enjeu, pour l'intervention ergonomique, est de savoir comment l'intervention fait histoire, comment telle « amélioration » transforme, ... comment le coup fait avancer la partie... « Les sciences humaines doivent traiter non seulement de la connexion des actions des hommes dans le monde, mais aussi de la constitution d'un monde de ces actions mêmes » (Salazar-Bondy, 1970). L'ergonomie a, là, une responsabilité de vigilance (Hubault, 1998) : faire la vigie c'est voir de plus haut et plus loin, c'est conduire ce qui se fait du point de vue dont ça agit, à partir des tendances qui se dessinent ou qui se ferment, en fonction des directions qui se découvrent. Ici, c'est donc le cadre et l'encadrement de l'intervention qui se trouvent exposés. Ce que Lévy appelle «perspectives d'intervention », le concept de « forces d'appel / de rappel sociales » (FARS) de Schwartz ${ }^{16}$. Rappelons que pour établir le périmètre de l'intervention qui permettra de l'évaluer, il faut pouvoir mettre en écho des référentiels épistémologiquement compatibles.

Nous retrouvons les enjeux de cette écologie à double face que nous avons proposée pour cadre de l'ergonomie : toute intervention gère la tension entre deux modes de constitution, la constitution de l'Entreprise comme milieu de travail et la constitution de l'Homme comme milieu de l'entreprise...

\section{Praxéologique :}

L'activité est une machine à tordre les concepts comme dit Schwartz. En interdisant toute prédiction réelle sur le travail réel, elle met en péril la lisibilité du métier des ergonomes, mais aussi le principe même de «science sociale ».

\section{- La lisibilité du «métier » des ergonomes}

Si l'activité est le moyen de leur pertinence dans les projets, et si elle pousse ce faisant les ergonomes à déborder les pointillés de ce qu'on croit leur domaine, il leur faut pouvoir faire face à l'environnement incrédule ou circonspect, mais aussi s'assurer eux-mêmes de la valeur de ce qu'ils font au regard des savoirs professionnels qu'ils peuvent tout autant menacer qu'enrichir. Tout autant donc pour asseoir leur position vis à vis de leurs clients que pour développer leur réputation vis-à-vis de leurs collègues, il faut une instance. Sans pratique réflexive, impossible de « mettre en patrimoine » ni de constituer le style en genre. Mais, sans dispositif approprié pour le faire, cette pratique est impossible. Ce dispositif est donc la condition nécessaire pour qu'une pensée réinjectable dans l'activité puisse se développer et développer ainsi la compétence professionnelle des intervenants. Car il est clair pour nous qu'on ne peut pas s'en tenir aux dispositifs « RH » habituellement en vigueur... La gestion de la filière métier dans les organisations par projets demande, pour les métiers de l'intervention (en interne comme en externe), d'être pensée en termes de réseau dépassant le cadre de la structure d'appartenance, dans une définition élargie de la communauté professionnelle. Le rôle des structures universitaires et de recherche, dans la mesure où elles seront clairement positionnées sur l'intervention comme question de recherche, nous apparaît de ce point de vue essentiel.

- Le principe même de "science humaine»

L'acte clinique peut se définir comme une intervention dans une situation toujours marquée par une crise du sens. Lévy (1997, p. 19) parle même d'une clinique $d u$ sens. Or, nous y avons déjà insisté : le sens n'est pas dans l'histoire, ni même dans son récit, mais dans sa reprise (Lévy, p. 24). En ergonomie donc, construire un problème c'est trouver le périmètre qui permet de comprendre comment l'activité est une synthèse dont on cherche à produire l'analyse pour en faire le levier d'un projet. Mais il faut pouvoir valider cette construction, la juger. Il faut donc l'arbitrage

16. Sous cet angle, la difficulté majeure vient du statut très singulier de l'économie qui contribue à lui conférer une sorte d'extraterritorialité : dans le monde « réaliste », l'économie organise les échanges mais elle n'échange elle-même contre rien : il n'y a pas d'équivalence de l'économie. C'est ce qu'il nous faut apprendre à dépasser (voir note supra). 
de ce que Schwartz nomme un "pôle d'exigences philosophiques ». Il prend soin de souligner qu'il ne faut pas le réduire au pôle des philosophes, organisant, dans des conjonctions nécessairement opportunistes, la rencontre entre disciplines qui ont en commun de s'intéresser à l'activité humaine $^{17}$... Installer et développer un tel dispositif nous semble incontournable si on veut pouvoir penser le développement de la recherche sur l'intervention, et donc le métier des chercheurs concernés par le développement de l'ergonomie comme (projet de) théorie de la transformation sociale par l'intervention dans les situations et les organisations de travail ${ }^{18}$.

Nous soutenons l'idée que l'originalité foncière de l'ergonomie tient au fait qu'elle configure à son propre niveau l'exigence du dispositif que Schwartz a proposé pour régler les rapports entre les « disciplines » concernées par l'activité humaine (Hubault, 1997). Il en a d'ailleurs convenu luimême, «l'ergonomie est peut-être aujourd'hui une propédeutique à l'épistémologie » (1996, p. 141).

Dès 1973 en effet, nous avons appris de Pierre Cazamian (Cazamian, Hubault \& Noulin, 1996) que le singulier est une synthèse et que la multidisciplinarité doit être pensée comme un dispositif à tonalité philosophique pour reconstituer et comprendre comment l'activité humaine dépasse dans l'action (cf. le « faire corps », " prendre corps »...) la discontinuité épistémologique des dimensions qu'elle engage. Ainsi, l'ergonomie relève d'entrée de ce type de dispositif dans lequel elle doit par ailleurs s'inscrire quand elle collabore avec d'autres disciplines « sœurs ».

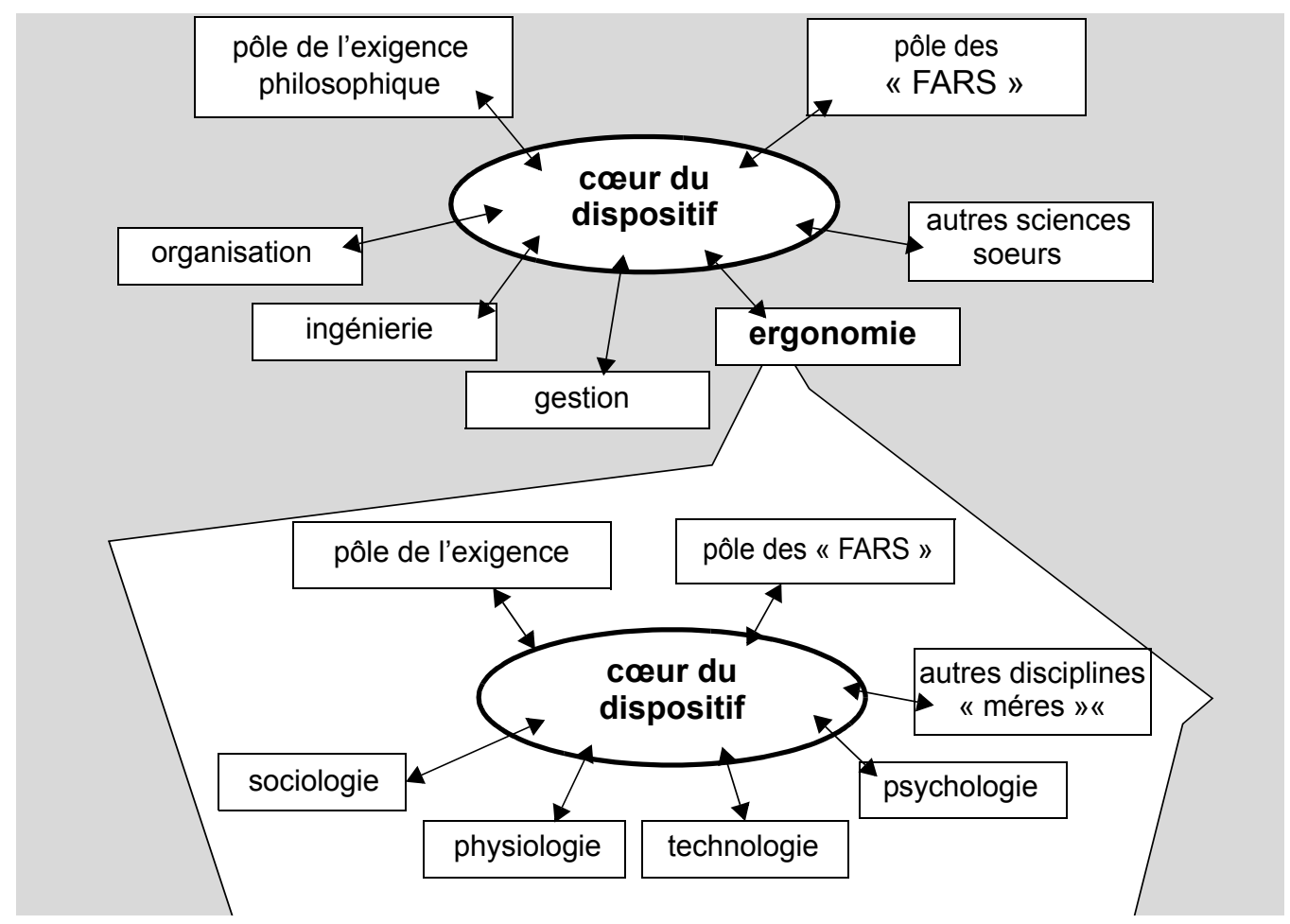

Figure 3.- le dispositif d'intervention de l'ergonomie.

17. C'est l'ambition même de l'ergologie : instruire cette rencontre entre les disciplines ayant l'activité humaine comme objet et enjeu commun : économie, droit, sociologie, psychologie, ... ergonomie...

18. Nous préférons ici cette périphrase, pour lourde qu'elle soit, au concept poppérien de « technologie sociale » aux connotations trop positivistes selon nous -même si une définition plus politique de la technologie existe aussi, on le sait ... 
D'entrée disions-nous ? Certes par intention et par vocation, mais il est plus exact de reconnaître que l'ergonomie a besoin de se doter d'un tel dispositif pour répondre à hauteur de son ambition.

Nous avons donc proposé d'étendre pour le compte propre de l'ergonomie (Figure 3), le schéma initialement développé par Schwartz à une échelle plus large, avec l'idée qu'en mettant en résonance ces deux niveaux de " divergence », on ouvre une conception stratégique nouvelle des modèles de changement dans les organisations qui doit permettre de penser la transformation qu'ils visent :

- concevoir l'ergonomie en termes de dispositif, c'est le moyen de dépasser les « sciences mères » et de proposer un autre cadre à l'analyse dont la divergence pourra être évaluée, et sa production progressivement " mise en patrimoine » au bénéfice d'un corps de connaissance disponible et transmissible. Pour paraphraser Clot, c'est le moyen d'assurer la «politique intérieure » de l'ergonomie ;

- pour paraphraser toujours Clot, la « politique extérieure » de l'ergonomie relève de la part du dispositif qui règle ses rapports avec les «disciplines sœurs»-parmi lesquelles la gestion et l'économie - dans les interventions qui requièrent de "croiser les regards " pour diverger ensemble;

- relier ces niveaux, enfin, cela ouvre une stratégie générale de recherche sur l'intervention dans les organisations ; c'est aussi la condition de développement du métier d'ergonome : l'exercice d'une pensée.

\section{BIBLIOGRAPHIE}

Baudrillard, J. (1997). Le paroxyste indifférent. Paris: Éditions Grasset.

Blazejewski, F., \& Hubault, F. (1999). De la gestion comme contexte à la gestion comme domaine, Conférence introductive au thème «Ergonomie et critères de gestion". Actes du 34ème congrès de la SELF. Caen, pp. 527-544.

Böhle, F., \& Milkau, B. (1998). De la manivelle à l'écran ; l'évolution de l'expérience sensible des ouvriers lors des changements technologiques. Paris: Éditions Eyrolles, coll. de la DER/EDF.

Bourgeois, F., Lemarchand, C., Hubault F., Brun, C., Polin, A., \& Faucheux J.-M. (2000). Travail et TMS. Quand la santé interroge l'organisation. Lyon: Editions Anact.

Cazamian, P., Hubault, F., \& Noulin, M. (1996). Traité d'ergonomie (nouvelle édition remaniée). Toulouse: Éditions Octarès.

Clot Y. (1995). Le travail sans l'homme? pour une psychologie des milieux de vie et de travail. Paris: Éditions La découverte.

Clot Y. (2000). La fonction psychologique du collectif. In Benchekroun T. H. et Weill-Fassina A. (coord), Le travail collectif, perspectives actuelles en ergonomie. Toulouse: Éditions Octarès, pp. 273-286.

Clot, Y., \& Faïta, Y. (2000). Genres et styles en analyse du travail. Concepts et méthodes, Revue Travailler, $\mathrm{n}^{\circ} 4$, pp. 7-42.

Daniellou, F. (Ed.) (1996). L'ergonomie en quête de ses principes, débats épistémologiques. Toulouse: Éditions Octarès.

Daniellou, F., \& Jackson, M. (1997). L'ergonomie intervient dans et sur des situations de gestion, Revue Performances, $\mathrm{n}^{\circ}$ Hors Série Coopérations et coordinations dans l'intervention en ergonomie; frontières, multidisciplinarité, collectifs d'action, séminaire Paris 1, Toulouse, pp. 16-20.

Dejours, C. (1995). Le facteur humain. Paris: PUF. Que sais-je nº2996.

Dejours, C. (1996). Épistémologie concrète et ergonomie. In (s/d) Daniellou F., L'ergonomie en quête de ses principes, débats épistémologiques. Toulouse: Éditions Octarès. pp. 201-217.

Girin, J. (1990). L'analyse empirique des situations de gestion : éléments de théorie et de méthode. In (s/d) Martinet, Épistémologies et sciences de gestion. Paris: Éditions Economica. 
Hubault, F. (1996). De quoi l'ergonomie peut-elle faire l'analyse ? In Daniellou F. (s/d), 1996, L'ergonomie en quête de ses principes, débats épistémologiques. Toulouse: Éditions Octarès. pp. 103-140.

Hubault, F. (1997). À quoi l'ergonomie participe-t-il ? Revue Performances Humaines et Techniques, $\mathrm{n}^{\circ}$ Hors Série Coopérations et coordinations dans l'intervention en ergonomie ; frontières, multidisciplinarité, collectifs d'action, Séminaire Paris 1, pp. 2-20.

Hubault, F. (1998). Pour une ergonomie de l'encadrement. Revue Performances Humaines et Techniques, $\mathrm{n}^{\circ}$ Hors Série Ergonomie de l'encadrement -pouvoirs et responsabilités des cadres, Séminaire Paris 1, Toulouse, pp. 2-9.

Latour, B. (1989). La science telle qu'elle se fait. Paris: Éditions Gallimard-Folio essais.

Lévy, A. (1997). Sciences cliniques et organisations sociales. Paris: PUF, coll. Psychologie sociale.

Magg,i B. (1996). La régulation du processus d'action de travail, in Cazamian, Hubault, Noulin, op. cit.

Mendel, G. (1998). L'acte est une aventure ; du sujet métaphysique au sujet de l'actepouvoir. Paris: Éditions La découverte.

Montmollin, M. (de), (2002). Discours sur l'organisation du travail. Paris: Éditions L'Harmattan, coll. Logiques sociales.

Popper, K. (1934, 1973). La logique de la connaissance scientifique. Paris: Éditions Payot.

Roustang, F. (2000). La fin de la plainte, Éditions Odile Jacob.

Salazar-Bondy, A. (1970). La science appliquée à l'homme peut-elle se passer d'axiologie ? In Axiologie et sciences de l'homme. Économies et Sociétés. Paris. Cahiers de l’ISEA (Éditions Droz), Tome IV, n¹2, pp. 2245-2254.

Schwartz, Y. (1996). Ergonomie, philosophie et extraterritorialité. In (s/d) Daniellou, L'ergonomie en quête de ses principes, débats épistémologiques, Toulouse: Éditions Octarès, pp. 141-182.

Schwartz, Y. (2000). Le paradigme ergologique ou un métier de philosophe. Toulouse: Éditions Octarès.

Waterson, P. E., \& Sell R. (à paraître), Recurrent theories and developments in the history of the Ergonomics Society. 